even more ways in which your university or school can help you to get through your day than you would think of in the first place. Without asking for help, you will never find out what is actually possible. Through communicating with a disabilityspokesperson you will most likely get even greater support.

In my lecture I will talk about my own story and how I managed to finish my Bachelor Degree whilst falling chronically ill with Adult Onset Still's Disease. I will give examples on how to handle a life with chronic illness. There will be some tips on what to do when your counterpart is not as understanding as he or she should be. I will explain what I do to get me through lectures and exams.

Disclosure of Interest: None declared

DOI: 10.1136/annrheumdis-2018-eular.7802

\section{SP0040 PARENTS SUPPORT FOR CHILDREN WITH RMDS IN EDUCATION}

M. Kepic. Society for Children with Immune Disease, Komenda, Slovenia

My presentation will contain situation in schools all across the Europe (at least 13 countries/results from a survey and info which I get from our Associations, members of ENCA).

It is serious situation. First I will present the problems, we, parents, are struggling with every day. I will also prepare and present possible solutions. One country is extra good in solutions and they really take good care for children with autoimunne conditions and we can learn from them. I will also present a concrete good solution.

Solutions should be used as a prepared document for all the countries. That is a goal of ENCA.

Disclosure of Interest: None declared

DOI: 10.1136/annrheumdis-2018-eular.7663

\section{THURSDAY, 14 JUNE 2018}

\section{WIN and HOT session}

\section{SP0041 WIN SESSION: WHAT IS NEW IN THE TREATMENT OF MYOSITIS?}

H. Chinoy ${ }^{1,2} .{ }^{1}$ National Institute for Health Research Manchester Biomedical Research Centre, Central Manchester University Hospitals NHS Foundation Trust, Manchester Academic Health Science Centre; ${ }^{2}$ The University of Manchester, Manchester, UK

Much progress has been made over the last decade in the understanding of the inflammatory myopathies. It is heartening to see some of these developments now in clinical practice which is hopefully increasing the quality of care for myositis patients. This talk will summary the new upcoming treatments in myositis. However, treatment of a rare disease not only involves administering pharmacotherapeutic agents, but recognising when to treat and which poor prognostic groups required intensification. Antibody testing of the spectrum of newly identified myositis serology now allows for better stratification of patients and assists the physician with treatment, monitoring and prognosis. New EULAR/ACR criteria are available for classification to assist inclusion into clinical trials, as well as agreed definitions for minimal, moderate and major response. A better understanding of the pathogenesis has now led to randomised controlled trials now being conducted in the inflammatory myopathies, including targeting the innate immune pathway that will hopefully lead to a licensed treatment for this difficult orphan disease.

Disclosure of Interest: H. Chinoy Grant/research support from: UCB, Novartis, Medlmmune - grant to University of Manchester, travel bursary - Abbvie, Janssen, Consultant for: Lilly, UCB, Janssen, Momenta, Novartis DOI: 10.1136/annrheumdis-2018-eular.7744

\section{SP0042 RECENT ADVANCES IN THE TREATMENT OF SYSTEMIC SCLEROSIS}

J.M. Van Laar. Rheumatology and Clinical Immunology, University Medical Centre Utrecht, Utrecht, Netherlands

Few rheumatological diseases have seen as many false dawns as systemic sclerosis (SSc) and many drugs once hailed as promising have fallen by the wayside. Clinical trials have been plagued by poor accrual, difficulties with definitions of endpoints, clinical heterogeneity of disease manifestations and variability of disease course, resulting in inconclusive or negative trial results. Clinicians standing in the shadows of no man's land could be forgiven for not seeing the sparks of light around them. Yet our grasp of the pathological pathways operative in SSc, consensus on endpoints and international collaboration have paved the way for clinical trials with novel antibrotic and immunomodulatory drugs (e.g. rociguat, JAK-STATi) that could change the way we treat SSc. For now we will have to stick to old friends such as methotrexate, MMF and cyclophosphamide in early diffuse cutaneous SSc, even though their clinical benefit is modest at best as shown in the ESOS study and expressed in recent guidelines. ${ }^{1-3}$ Rituximab is increasingly being used based on observational studies in SSc and its proven potency in rheu matoid arthritis, but requires more robust data. Tocilizumab has shown unexpected benefit on lung function in a phase 2 trial, ${ }^{4}$ which if confirmed with phase 3 trial could transform the way we manage SSc-ILD. As of yet, the only proven disease modifyinge therapy in early progressive SSc is autologous hematopoietic stem cell transplantation, shown to be effective in two prospective, controlled randomised clinical trials (ASTIS and SCOT, conducted in Europe and North America respectively) $\left(.^{5,6}\right.$ Only a small group of patients qualify for this howeve and even fewer have access to centres with the necessary expertise. SSc continues to constitute an area of unmet need, yet progress is being made, albeit slow.

\section{REFERENCES :}

[1] Herrick, et al. Ann Rheum Dis 2017;76:1207-18.

[2] Kowal-Bielecka, et al. Ann Rheum Dis 2017;76:1327-39.

[3] Denton, et al. Rheumatology 2016;55:1906-10.

[4] Khanna, et al. Ann Rheum Dis 2018;77:212-20.

[5] Van Laar, et al. JAMA 2014;311:2490-8.

[6] Sullivan, et al. N Engl J Med 2018;378:35-47.

Disclosure of Interest: J. Van Laar Grant/research support from: Astra Zeneca, Roche-Genentech, MSD, Consultant for: Arthrogen, BMS, Eli Lilly, Pfizer, Roche, Speakers bureau: Janssen

DOI: 10.1136/annrheumdis-2018-eular.7779

THURSDAY, 14 JUNE 2018

Can we halt progression of structural damage in axial SpA?

\section{SP0043 HOW TO CAPTURE CLINICALLY RELEVANT STRUCTURAL PROGRESSION IN AXIAL SPA}

\section{Van Der Heijde. Leiden University Medical Center, Leiden, Netherlands}

Structural damage in axial SpA usually refers to the sacro-iliac (SI) joints or the spine. The classic order of the progress of structural damage is in the SI joints, followed by the spine. Consequently, in early disease assessment of structural damage should be focused on the SI joints and in established disease (i.e. in patients with radiographic sacroillitis) evaluation of the spine becomes more useful.

The most striking abnormality representing structural damage in axial $\mathrm{SpA}$ is bone formation, although in early phases bone destruction can also be seen. Typically, structural damage is assessed on radiographs.

For radiographs there are two frequently used scoring methods: grading of the S joints according to the modified New York (mNY) criteria and assessment of the spine by the modified Stoke Ankylosing Spondylitis Score (mSASSS). The mNY grades range from 0-4 per SI joint, 0-8 in total. Although it is well known that reliability of $\mathrm{mNY}$ grading is poor, this continuous grading has recently been successfully used as an outcome measure. The mSASSS is mainly based on bone formation in the anterior vertebral corners in the cervical and lumbar spine (range $0-72$ ). It is a reliable measure but the progression observed by mSASSS is rather modest, resulting in a minimum follow-up of two years.

Imaging of structural damage by MRI is another option. Due to the imaging of the whole spine and the three-dimensional technique this seems an attractive alternative. However, especially bone formation is very hard to assess. Fatty deposition is a typical MRI abnormality, which can be seen as an intermediate step between inflammation visible on MRI and bone formation on radiographs. However, this finding is insufficiently validated to be able to consider it as a true surrogate for structural damage.

Finally, a CT scan is a method having the advantages of MRI (whole spine, tomographic technique) but also the best capabilities of bone imaging. The big disadvantage of CT scan is the radiation dose. Recently, it became possible to make images with good image quality but acceptable radiation, the so-called low-dose CT. A validated scoring system for CT exists: the CT scoring system (CTSS). Only bone proliferation is assessed in this method. In a direct comparison with mSASSS more progression was observed over a 2 year period. This was mainly due to the progression observed in the thoracic spine.

Only structural damage assessed by the mSASSS has shown a clear relationship with outcomes that are important for patients such as function and quality of life. No data on MRI or CTSS exist. However, it is hard to describe what defines a clinically relevant progression. The interval to assess progression to assess a 
treatment effect is typically 2 years. Only small changes can be assessed over this period, normally about 1-2 mSASSS units. It is hard to define that this is clinically relevant. The most important for treatment is to show that there is inhibition of structural progression in comparison to untreated patients, especially as axial $\mathrm{SpA}$ is a lifelong disease and 1 unit over 30 years still leads to severe ankylosis of the spine.

Disclosure of Interest: None declared

DOI: 10.1136/annrheumdis-2018-eular.7656

\section{SP0044 WHAT DO WE LEARN FROM RCTS ON THE TREATMENT EFFECT ON STRUCTURAL PROGRESSION IN AXSPA?}

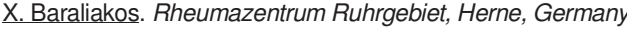

The introduction of tumour necrosis factor inhibitors (TNFis) about 20 years ago has led to the hope of disease modification of ankylosing spondylitis, since biologics showed for the first time a decrease of inflammatory activity on MRI, with the latter being theoretically also directly linked to new bone formation. However, the first open-label extensions of randomized-controlled trials with a treatment duration of 2 years failed to show any positive effect on the radiographic progression in AS patients when compared to historical cohorts that had not been exposed to biologics. Nevertheless, later data indicated that this lack of influence on radiographic progression might have been due to many different reasons that were not taken into account in these first analyses, such as the radiographic status of patients at baseline, CRP levels or insufficient duration of follow-up. Furthermore, most recent data from MRI studies also indicated that the most important link to influence radiographic progression with biologics might not be the suppression of inflammation but the protection of bone to show tissue metaplasia to post-inflammatory findings, while early suppression of inflammation might be the key to even completely inhibit radiographic progression in AS patients.

Indeed, most recent cohort data have been able to demonstrate an association between TNF-blocker treatment and reduced risk of spinal structural progression (e.g. formation of syndesmophytes). Furthermore, early escalation of treatment from NSAIDs to biologics and long-term treatment with biologics have also independently been able to show positive effects on radiographic progression in patients with AS. Finally, also newer biologics such as IL-17A inhibitors have also provided promising results in terms of overall low radiographic progression rates as measured by validated scoring systems.

Currently, first head-to-head trials of different biologics are underway to examine any possible differences between the available compounds with a primary outcome of their effect on spinal radiographic progression.

It remains to be shown whether and how these results will also become clinically relevant in terms of decrease or even inhibition of spinal mobility restrictions, in order to be able to postulate a 'real' disease modifying effect of biologic treatment in axial spondyloarthritis.

Disclosure of Interest: None declared

DOI: 10.1136/annrheumdis-2018-eular.7840

\section{THURSDAY, 14 JUNE 2018}

\section{Reproductive issues in rheumatology}

\section{SP0045 OESTROGENS, IMMUNE RESPONSE AND AUTOIMMUNE DISEASES}

M. Cutolo, on behalf of Eular Study Group on Neuroendocrinelmmunology of the Rheumatic Diseases. Research Lab. Division Rheumatology. Dept Internal Medicine University of Genova Italy, genova, Italy

Sex hormones are implicated in the immune response, with estrogens as enhancers at least of the humoral immunity and androgens and progesterone (and glucocorticoids) as natural immune-suppressors. Several physiological, pathological and therapeutic conditions may change the serum oestrogen milieu including the menstrual cycle, pregnancy, postpartum period, menopause, elderly, chronic stress, altered circadian rhythms, inflammatory cytokines, use of glucocorticoids, oral contraceptives, and steroid hormonal replacements. Cortisol and melatonin circadian rhythms are altered, at least in rheumatoid arthritis (RA), and partially involve also sex hormone circadian synthesis and levels. Abnormal regulation of aromatase activity (i.e. increased activity) by inflammatory cytokines production (i,e, TNF-alpha, IL-1, IL-6) may partially explain the abnormalities of peripheral oestrogen synthesis in RA (i.e. increased availability of 17-beta estradiol and possible metabolites in synovial fluids) and in systemic lupus srythematosus (SLE). In the synovial fluids of RA patients the increased oestrogen concentration are observed in both sexes and are more specifically characterised by the hydroxylated forms, in particular 16alpha-hydroxyestrone, that is a mitogenic and cell proliferative endogenous hormone. Local effects of sex hormones in autoimmune rheumatic diseases seems to consist mainly in modulation of cell proliferation.

Epidemiological evidence indicates that during the fertile age women are more often affected by rheumatic diseases than men, particularly autoimmune diseases. As a matter of fact, rheumatic disorders with autoimmune involvement such as RA or SLE, result from the combination of several predisposing factors, that include the relationships between epitopes of the trigger agent (i.e. virus), the status of the stress response system including the hypothalamic-pituitary-adrenocortical axis (HPA) and mainly the effects of the gonadal hormones (hypothalamic-pituitary-gonadal axis - HPG)

The pre-or post-menopausal serum sex hormonal status is a further factor influencing the rate of rheumatic diseases. It is therefore important, whenever possible, to evaluate epidemiologic data broken down into age (for example 10 year age band) and sex-specific group before making inferences. Obviously, sex hormones seem to play an important role as modulators of both disease onset and perpetuation and show circadian rhythms together with cortisol.

Sex hormones are implicated in the immune response, with estrogens as enhancers at least of the humoral immunity and androgens and progesterone (and glucocorticoids) as natural immune-suppressors. Low concentrations of gonadal and adrenal androgens [testosterone ( $\mathrm{T})$ /dihydrotestosterone (DHT), dehydroepiandrosterone (DHEA) and its sulphate (DHEAS), respectively] levels, as well as reduced androgens/estrogens ratio, have been detected in serum and body fluids (i.e. blood, synovial fluid (SF), smears, salivary) of male and female RA patients, as well as in SLE, supporting the possible pathogenic role for the decreased levels of the immune-suppressive androgens. However, respect to serum levels of estrogens, interestingly they are not significantly changed which is in strict contrast to androgen levels in RA patients (reduced).

As a matter of fact, sex hormones can exert also local actions (paracrine) in the tissues in which they are formed or enter the circulation and both $T$ and 17-beta estradiol seem to exert dose and time-dependent effects on cell growth and apoptosis. These effects, as well as important influences on gene promoter of Th1/Th2 cytokines and the recently discovered increased SF oestrogen concentrations might suggest new interesting roles for estrogens at least in RA. Finally estrogens exert important epigenetic actions on cell proliferation. Estrogens act as key fac tors in cellular proliferation and differentiation as well as cancer development and progression (prostate). The expression of oestrogen receptor (ER)- $\beta$ appears to be lost during prostate cancer progression through hypermethylation mechanism Epigenetic drugs such as 5-aza-2'-deoxycytidine (5-AZAC) and Trichostatin A (TSA) showed efficacy in restoring ER $\beta$ expression in prostate cancer cells These observations highlights that the strategy of merging epigenetic and hormonal therapies might be beneficial also in inflammatory/autoimmune diseases (synovial tissue)

\section{REFERENCES}

[1] Cutolo M, et al. Estrogen's effects in chronic autoimmune/inflammatory dis eases and progression to cancer. Expert Rev Clin Immunol 2014;10:31-9.

[2] Cutolo M, et al. Ann. Rheum. Dis 2005;64:212-6.

[3] Cutolo M, et al. Autoimmun. Rev 2005;4:497-502.

[4] Cutolo M, et al. Ann. N. Y. Acad. Sci 2005;1051:372-8.

[5] Janele D, et al. Ann. N. Y. Acad. Sci 2006;1069:168-82.

[6] Motawi TK, et al. Life Sci 2018 Feb 15;pii: S0024-3205(18)30069-9

Disclosure of Interest: None declared

DOI: 10.1136/annrheumdis-2018-eular.7725

\section{SP0046 \\ WHAT WE NEED TO CONSIDER IN PHYSICIAN-PATIENT COMMUNICATION ON SEXUAL PROBLEMS IN DIFFERENT RHEUMATIC CONDITIONS}

M. Ostensen. Department of Rheumatology, St.Olavs Hospital, Kristiansand, Norway

Quality of life (QOL) is often reduced in patients with chronic diseases. Sexual activity and enjoyment constitute an important aspect of QOL. Sexuality is a neglected area of QOL in patients with rheumatic disease.

Sexual problems among patients are common and often increase with disease duration. Both disease related factors and the psychological response to chronic disease can impair sexual functioning. General disease symptoms like pain, fatigue, disease activity, and impaired physical function contribute to reduced sexual activity in both genders. However, psychological factors like depression, anxiety, negative body image and low self-esteem play an important role. Sexua dysfunction can create frustration and distress, and if chronic increase anxiety and depression, and damage interpersonal relationships. 